\title{
ANÁLISE DA VALIDADE DE CONSTRUCTO DO INSTRUMENTO DE CLASSIFICAÇÃO DE PACIENTES PROPOSTO POR PERROCA
}

\author{
Marcia Galan Perroca ${ }^{\top}$ \\ Raquel Rapone Gaidzinski
}

Perroca MG, Gaidzinski RR. Análise da validade de constructo do instrumento de classificação da pacientes proposto por Perroca. Rev Latino-am Enfermagem 2004 janeiro-fevereiro; 12(1):83-91.

Este artigo parte de uma pesquisa sobre a avaliação da confiabilidade e validade do instrumento de classificacão de pacientes, elaborado por Perroca, e tem por propósito analisar a validade de constructo desse instrumento, bem como verificar sua aplicabilidade na prática gerencial do enfermeiro. Integraram o estudo 141 pacientes, alocados em Unidades de Internacão e Unidades de Terapia Intensiva, de um hospital de ensino no interior de São Paulo. A validade de constructo do instrumento foi realizada por um conjunto de tratamentos estatísticos. O resultado do estudo evidenciou todos os indicadores críticos como importantes, tendo cada um papel próprio na determinação do grau de complexidade do paciente em relação à atenção de enfermagem, assim como mostrou que o instrumento apresenta evidências de validade, estando em condições de ser aplicado na prática gerencial do enfermeiro como norteador das necessidades de cuidado do paciente, bem como da carga de trabalho da equipe de enfermagem.

DESCRITORES: avaliação em enfermagem; validade; cuidado de enfermagem

\section{PERROCA'S PATIENT CLASSIFICATION INSTRUMENT: CONSTRUCT VALIDITY ANALYSIS}

This paper starts from a research on the reliability and validity assessment of Perroca's patient classification instrument. Its purpose is to analyze the construct validity of this instrument, as well as to check its use in nursing management practice. Study participants were a hundred forty-one patients, allocated in Intensive Care and Hospitalization Units of a school hospital in the interior of São Paulo State, Brazil. Several statistical techniques were used to show the construct validity of the instrument. Research results revealed the importance of all critical indicators, each of which has its own role in determining the patient's complexity degree in relation to nursing care. Therefore, the study showed that the instrument shows evidence of validity. Hence, it is useful for nursing management practice, as a guide to the patient's care needs as well as to the nursing team's workload.

DESCRIPTORS: nursing assessment; validity; nursing care

\section{ANÁLISIS DE LA VALIDAD DE CONSTRUCTO DEL INSTRUMENTO DE CLASIFICACIÓN DE PACIENTES PROPUESTO POR PERROCA}

Este artículo, parte de una investigación sobre la evaluación de la confiabilidad y validad del instrumento de clasificación de pacientes elaborado por Perroca, tiene por objetivo analizar la validad de constructo de este instrumento, así como averiguar su aplicabilidad en la práctica gerencial del enfermero. Integraron el estudio 141 pacientes ubicados en Unidades de Vigilancia y Unidades de Terapia Intensiva de un hospital de enseñanza en el interior de São Paulo. La validad de constructo del Instrumento se realizó por medio de un conjunto de tratamientos estadísticos. El resultado evidenció todos los indicadores críticos como importantes, teniendo cada uno papel propio en la determinación del grado de complejidad del paciente con respecto a la atención de enfermería, así como que el instrumento presenta evidencias de validad estando en condiciones de aplicarse en la práctica gerencial del enfermero como guía de las necesidades de cuidado del paciente, así como de la carga de trabajo del equipo de enfermería.

DESCRIPTORES: evaluación en enfermería; validez; atención cuidado de enfermería

\footnotetext{
${ }^{1}$ Doutor em Enfermagem, Docente da Faculdade de Medicina de São José do Rio Preto, e-mail: marciaperroca@telia.com; ${ }^{2}$ Professor Livre Docente da Escola de Enfermagem da Universidade de São Paulo, Orientador
} 
INTRODUÇÃO

Este artigo é parte de uma tese de doutorado conduzida com o intuito de monitorar a confiabilidade e a validade do instrumento de classificação de pacientes, elaborado por Perroca ${ }^{(1)}$, Perroca e Gaidzinski ${ }^{(2)}$, baseado nas necessidades individualizadas de cuidado de enfermagem. Os instrumentos de classificação são utilizados para categorizar pacientes de acordo com a quantidade de cuidado de enfermagem requerida, ou seja, baseado no grau de complexidade da assistência de enfermagem.

O conceito de validade é abordado por diversos autores como sendo o grau em que um instrumento mostra-se apropriado para mensurar o que supostamente ele deveria medir ${ }^{(3-6)}$. Assim, quando se submete um instrumento ao procedimento de validação, na realidade não é o instrumento em si mesmo que está sendo validado, mas sim o propósito pelo qual o instrumento está sendo usado $^{(4,6-7)}$.

A determinação da validade é facilitada quando múltiplas medidas são empregadas para responder a uma dada questão da pesquisa. A convergência de resultados, obtidos quando do uso de variadas técnicas, possibilita aumento da confiabilidade e validade dos achados do estudo e sua utilização na prática, uma vez que eles se encontram solidamente apoiados em evidências ${ }^{(5,7)}$.

Existe uma variedade de enfoques para se viabilizar a demonstração da validade de um instrumento, contudo, nesta pesquisa, utilizaremos apenas a abordagem relativa à validade de constructo. Esse tipo de validade, também denominada de validade de conceito, é apontada como complexa e desafiadora para o pesquisador. O desafio encontra-se em se descobrir se a representação (teste) constitui uma representação legítima e adequada do constructo. Pode-se considerar constructo como conceito que foi deliberadamente criado, ou adotado, para um determinado propósito científico ${ }^{(3,8)}$.

A validade de constructo pode ser acessada quando o pesquisador acredita que o seu instrumento reflete um constructo particular que está atrelado a certos significados. A interpretação proposta vai gerar hipóteses significativas que, testadas, vão fornecer um significado de confiança, ou não, referente ao que foi alegado ${ }^{(6)}$. $O$ constructo que se pretende acessar, nesta investigação, é o grau de complexidade do paciente.

Dessa forma, para se afirmar que um instrumento para classificação de pacientes é válido, é necessário que ele passe por um processo que permita concluir se os indicadores de cuidado representam, de fato, o que é encontrado na prática clínica. Isso implica em alguns questionamentos tais como: em que extensão o instrumento consegue medir com precisão a necessidade de cuidado do paciente com relação à enfermagem? Estariam os indicadores de cuidado em número suficiente? Diante do exposto, esta investigação tem por propósito analisar a validade de constructo do instrumento de classificação de pacientes, proposto por Perroca.

\section{TRAJETÓRIA METODOLÓGICA}

O instrumento de classificação de pacientes elaborado por Perroca ${ }^{(1)}$ é composto por 13 indicadores críticos de cuidados (necessidades de cuidado de enfermagem do paciente): Estado Mental e Nível de Consciência, Oxigenação, Sinais Vitais, Nutrição e Hidratação, Motilidade, Locomoção, Cuidado Corporal, Eliminações, Terapêutica, Educação à Saúde, Comportamento, Comunicação e Integridade CutâneoMucosa. Cada um dos indicadores possui gradação de 1 a 5, apontando a intensidade crescente de complexidade do cuidado, de forma que, o valor 1 corresponde ao menor nível de complexidade assistencial e o valor 5, ao nível máximo de complexidade assistencial. O paciente é classificado em todos os indicadores, em um dos 5 níveis, na opção que melhor descreva a sua situação em relação à assistência de enfermagem. O valor obtido individualmente, em cada um dos indicadores, é somado e o valor total obtido é comparado com os intervalos de pontuações propostos, conduzindo, dessa forma, a uma classe, ou categoria de cuidado, a que esse paciente pertence: cuidados mínimos (13-26 pontos), cuidados intermediários (27-39 pontos), cuidados semi-intensivos (40-52 pontos) e cuidados intensivos (53-65 pontos).

Com o intuito de estabelecer a validade de constructo desse instrumento foram utilizados os conjuntos dos seguintes tratamentos estatísticos: Análise de Componentes Principais (ACP), Análise de Variância e o Coeficiente de Correlação de Pearson.

A ACP é uma técnica de transformação de variáveis que, a partir de um conjunto de $\mathbf{p}$ variáveis de interesse, gera um novo conjunto de $\mathbf{p}$ variáveis (denominadas componentes principais) de tal forma que 
a primeira componente é aquela que apresenta maior variância possível dentre todas as possíveis combinações lineares das variáveis originais e as demais componentes recebem uma ordenação em função de sua variabilidade ${ }^{(9-}$ 10). A Análise de Variância (ANOVA) constitui-se em um procedimento estatístico utilizado para testar a significância das diferenças entre as médias de três ou mais grupos. Constitui-se em teste de hipótese para verificar com precisão os resultados e seu afastamento da expectativa baseada no acaso ${ }^{(4,7,11)}$.

O procedimento de correlação constitui-se no método mais comum para se descrever a associação entre variáveis quantitativas. Os coeficientes de correlação são, normalmente, designados com um $\mathbf{r}$ e costumam variar de $+1,00$ (para uma relação direta perfeita), passando por 0,00 (ausência de relação) e chegando a $-1,00$ (para uma relação inversa perfeita). O coeficiente de correlação mais usado, designando a magnitude de relação entre duas variáveis, mensuradas em pelo menos uma escala por intervalo é o chamado coeficiente de correlação, ou $\mathbf{r}$ de Pearson $^{(7)}$.

A validação clínica do instrumento de classificação de pacientes foi realizada nas Unidades de Internação e nas Unidades de Terapia Intensiva de um hospital de ensino, de grande porte, no interior do Estado de São Paulo. Foi utilizada, nesse estudo, uma amostragem estratificada por partilha proporcional, correspondente a $25 \%$ do total dos pacientes internados nas unidades de Clínica Médica, Clínica Cirúrgica, Clínica Ginecológica e Obstétrica e Unidades de Terapia Intensiva (excluídas as Terapias Intensivas Pediátrica e Neonatal), escolhidos por um procedimento sistemático, perfazendo um total de 141 pacientes.

No que diz respeito aos aspectos éticos envolvidos na realização desta pesquisa, cumpre esclarecer que a coleta de informações somente foi iniciada após parecer favorável do Comitê de Ética em Pesquisa da instituição campo de estudo. As enfermeiras responsáveis pelas unidades envolvidas nesta investigação foram orientadas quanto aos objetivos, etapas da investigação e forma como atuariam na mesma, bem como sobre a estrutura básica do instrumento e a sua forma de operacionalização. $O$ instrumento foi testado durante 5 dias consecutivos, no plantão da manhã, nas unidades citadas anteriormente, sempre pelas mesmas enfermeiras.

Os dados obtidos dos formulários foram processados estatisticamente em computador, utilizando- se o programa Minitab, versão 12.2. Para viabilizar a demonstração da validade de constructo do instrumento de classificação proposto por Perroca ${ }^{(1)}$, recorreu-se às múltiplas técnicas de tratamento de dados que possibilitassem responder às inquirições da pesquisadora. Dessa forma, para se determinar a possibilidade, ou não, de se diminuir o número de indicadores críticos de cuidado que compõem o instrumento, optou-se pela Análise de Componentes Principais e Análise de Variância; e, para se observar o comportamento das variáveis, ou seja, dos 13 indicadores críticos de cuidado e estudar a associação entre elas, utilizou-se o Coeficiente de Pearson.

\section{RESULTADOS E DISCUSSÃO}

Apresentamos, a seguir, os resultados obtidos mediante a utilização da Análise dos Componentes Principais, Análise de Variância e Coeficiente de Pearson entre os indicadores críticos, em tabelas e figuras, iniciando pela caracterização dos pacientes avaliados pelas enfermeiras e a classificação em categorias de cuidados por unidades.

A faixa etária dos pacientes estudados oscilou entre 13 e 89 anos, com maior incidência de pacientes nos grupos etários de 51 a 70 anos. Dos 141 pacientes avaliados pelas enfermeiras, durante este estudo, 92 (65,2\%) foram categorizados como de Cuidados Mínimos; $32(22,7 \%)$ como sendo de Cuidados Intermediários; 6 $(4,3 \%)$ como Cuidados Semi-Intensivos e 11 (7,8\%) como de Cuidados Intensivos. Nas unidades de Clínica Médica, Cirúrgica e Ginecoobstétrica houve predominância de pacientes de cuidados mínimos. Contudo, as Unidades de Terapia Intensiva Geral e Cardiológica, apesar de apresentarem elevado percentual de pacientes que exigem nível de atenção de enfermagem intensivo (50\%) como é esperado, também revelaram um número expressivo de pacientes de cuidados mínimos (30\%) e intermediários (20\%).

Análise de Componentes Principais (ACP) e Análise de Variância

Foi efetuada a Análise de Componentes Principais para estudar as associações entre os 13 indicadores e seus efeitos na discriminação dos pacientes entre si. Essa análise aponta os fatores que mais determinam as 
diferenças entre os indivíduos. Os fatores podem ser considerados como variáveis latentes, as quais não são observáveis, mas regulam as diferenças interindividuais no grupo observado.

A ACP foi feita sobre a matriz de covariâncias. Esses fatores são hierarquizados por capacidade de explicação da variação total, medida global de dispersão entre os indivíduos observados, representada pela proporção explicada da variação total. Os primeiros explicam, conjuntamente, a proporção acumulada da variação total. A Tabela 1 apresenta a análise de componentes principais dos fatores 1, 2, 3, 11, 12 e 13, mostrando quanto cada fator explica e o quanto cada variável entra na composição dos fatores através dos pesos fatoriais.

Tabela 1 - Apresentação dos pesos fatoriais da Análise de Componentes Principais. São José do Rio Preto, SP, 2000

\begin{tabular}{|c|c|c|c|c|c|c|}
\hline \multicolumn{7}{|c|}{ Matriz de Covariância } \\
\hline Autovalor & 14,665 & $5 \quad 1,822$ & 1,128 & 0,230 & 0,214 & 0,156 \\
\hline Proporção & 66,7 & 0,083 & 0,051 & 0,010 & 0,010 & 0,007 \\
\hline Cumulativo & 0,667 & 0,750 & 0,801 & 0,983 & 0,993 & 1,000 \\
\hline $\begin{array}{l}\text { Variável } \\
\text { IND } 1\end{array}$ & $\begin{array}{c}\text { PC1 } \\
0,250\end{array}$ & $\begin{array}{r}\text { PC2 } \\
-0,313\end{array}$ & $\begin{array}{c}\text { PC3 } \\
-0,213\end{array}$ & $\begin{array}{l}\text { PC11 } \\
-0,386\end{array}$ & $\begin{array}{l}\text { PC12 } \\
0,476\end{array}$ & $\begin{array}{c}\text { PC13 } \\
-0,462\end{array}$ \\
\hline IND 2 & 0,224 & $-0,269$ & 0,162 & 0,072 & $-0,283$ & $-0,230$ \\
\hline IND 3 & 0,125 & $-0,085$ & 0,494 & $-0,015$ & 0,067 & 0,192 \\
\hline IND 4 & 0,301 & $-0,106$ & 0,158 & 0,003 & $-0,410$ & $-0,195$ \\
\hline IND 5 & 0,333 & 0,274 & $-0,202$ & 0,360 & $-0,124$ & 0,052 \\
\hline IND 6 & 0,360 & 0,423 & $-0,041$ & $-0,716$ & $-0,268$ & 0,053 \\
\hline IND 7 & 0,363 & 0,327 & $-0,189$ & 0,376 & 0,319 & $-0,148$ \\
\hline IND 8 & 0,352 & 0,295 & 0,086 & 0,140 & 0,051 & $-0,025$ \\
\hline IND 9 & 0,251 & $-0,026$ & 0,673 & 0,011 & 0,356 & 0,071 \\
\hline IND 10 & 0,168 & $-0,231$ & 0,015 & 0,085 & $-0,319$ & 0,263 \\
\hline IND 11 & 0,262 & $-0,406$ & $-0,159$ & 0,140 & $-0,212$ & $-0,188$ \\
\hline IND 12 & 0,300 & $-0,287$ & $-0,306$ & $-0,106$ & 0,209 & 0,722 \\
\hline IND 13 & 0,189 & $-0,249$ & $-0,054$ & 0,064 & 0,110 & $-0,007$ \\
\hline
\end{tabular}

$\mathrm{Na}$ análise acima, com todos os 13 indicadores críticos, os 2 primeiros fatores explicam $75 \%$ da variação total dos dados. O primeiro fator, por si só, representa $66,7 \%$ da variabilidade total, sendo considerado, dessa forma, o mais importante, aquele que tem mais poder de discriminação entre os indivíduos. Quando analisamos os demais fatores é possível notar que a quantidade de explicação cai demasiadamente, do primeiro fator $(66,7 \%)$ para o segundo fator $(8,3 \%)$. Do fator 2 para o fator 3 , caem 3 pontos percentuais, do fator 3 para 04 na base de 1 ponto percentual e a queda percentual vai diminuindo.

Como a ordenação dos fatores é por ordem de importância e a capacidade de explicação dos fatores de 3 a 13 é muito baixa, serão considerados nesta análise apenas os fatores 1 e 2 . Os demais fatores não serão explorados por terem menor explicação. Observando-se os dois primeiros fatores acima, verifica-se que o primeiro deles representa um conceito de "gravidade global", isto é, separa os indivíduos em categorias de cuidados mínimos, intermediários, semi-intensivos e intensivos, ordenando-os (Figura 1). Esse primeiro fator aponta todos os indicadores críticos como sendo indicadores de gravidade, pois nenhum indicador apresenta peso próximo de zero. Isso significa valorização de todos os indicadores críticos que compõem o instrumento como discriminadores do grau de complexidade do paciente.

É importante questionar, contudo, quais são os indicadores críticos que mais contribuem para explicar o fator 1 , ou seja, quais são os indicadores críticos mais envolvidos na percepção da mudança de categoria de cuidado. Existe uma técnica para se verificar o grau de importância de uma variável (indicador crítico) no fator estudado. Essa técnica consiste em se multiplicar o valor máximo obtido em uma das variáveis do fator por 0,7. Em nossa situação, se multiplicarmos 0,363 (valor máximo obtido no indicador 7) por 0,7 obteremos o valor 0,254, o qual representa o limite máximo, na interpretação fatorial, significando que há um envolvimento da variável (indicador crítico) naquele fator.

Se observarmos os pesos fatoriais é possível destacar que os indicadores realmente significantes no fator 1 são: 1 (Estado Mental e Nível de Consciência), 4 (Nutrição e Hidratação), 5 (Motilidade), 6 (Locomoção), 7 (Cuidado Corporal), 8 (Eliminações), 9 (Terapêutica), 11 (Comportamento) e 12 (Comunicação). O indicador crítico 7 (Cuidado Corporal) é aquele que apresenta maior peso fatorial, ou seja, é indicador mais importante para captar a mudança na categoria do cuidado. Estudo realizado no Instituto do Coração da Faculdade de Medicina da Universidade de São Paulo, para classificar os pacientes internados na Unidade de Recuperação Cardíaca, mostrou, igualmente, que o indicador mais significativo na determinação da complexidade assistencial referia-se ao cuidado corporal $^{(12)}$.

Os indicadores 2 (Oxigenação), 3 (Sinais Vitais), 10 (Educação à Saúde) e 13 (Integridade Cutâneo-Mucosa) podem ser considerados como não muito importantes, ou seja, não estão muito envolvidos nesse fator. O indicador 3 (Sinais Vitais) é o que apresenta menor peso fatorial $(0,125)$, demonstrando, dessa forma, menor importância na determinação da mudança da categoria de cuidado.

O segundo fator, com $8,3 \%$ de explicação da variação total, mostra uma contraposição entre cuidados 
extremos (mínimos e intensivos) contra os cuidados intermediários e semi-intensivos. Pode-se dizer que esse fator separa os pacientes com maiores notas obtidas nos indicadores 5 (Motilidade), 6 (Locomoção), 7 (Cuidado Corporal) e 8 (Eliminações) e notas menores obtidas nos indicadores 1 (Estado Mental e Nível de Consciência), 2 (Oxigenação), 10 (Educação à Saúde), 11 (Comportamento), 12 (Comunicação) e 13 (Integridade Cutâneo-Mucosa) daqueles pacientes com valoração invertida nos mesmos indicadores, isto é, onde a nota era maior passa a ser menor e vice-versa.

Os indicadores 1 (Estado Mental e Nível de Consciência), 6 (Locomoção), 7 (Cuidado Corporal) e 11 (Comportamento) são aqueles que mais contribuem na percepção dessa contraposição interna dentro dos grupos de categorias de cuidados. É ainda possível observar que os indicadores 3 (Sinais Vitais), 4 (Nutrição e Hidratação) e 9 (Terapêutica) são pouco expressivos nessa ação.

A característica do fator 2 é que ele aponta diferenças entre os pacientes dentro de cada categoria de cuidados (Figura 1). Ele revela contraposição entre uma combinação dos indicadores Motilidade, Locomoção, Cuidado Corporal e Eliminações e uma combinação dos indicadores críticos Estado Mental e Nível de Consciência, Educação à Saúde, Comportamento e Comunicação. Isso pode ser evidenciado pelos sinais positivos e negativos dos pesos fatoriais desses indicadores críticos.

Se analisarmos a trajetória dos indicadores críticos 3 (Sinais Vitais), 9 (Terapêutica) e 13 (Integridade Cutâneo-Mucosa), ao longo dos 13 fatores, podemos constatar que apesar de eles perceberem o grau de complexidade do cuidado no primeiro fator, eles não são muito expressivos se comparados aos demais indicadores críticos. O indicador 3 (Sinais Vitais) é o que apresenta menor peso fatorial no primeiro fator $(0,125)$, valores insignificantes no segundo fator $(0,085)$ e um valor importante no terceiro fator $(0,494)$. Contudo, esse último fator explica apenas 5,1\% da variação total. O indicador 9 (Terapêutica) está no limiar de importância, com base nos $70 \%$ do peso máximo dado pelo fator $1(0,251)$. Mas, no fator 2 , ele não pesa quase nada $(0,026)$. O indicador 13 (Integridade Cutâneo-Mucosa) é pouco importante no fator $1(0,189)$, passando a ter maior significância no fator 4 $(0,776)$, o qual explica apenas $3,9 \%$ da variação total.

Pode-se concluir, frente aos resultados obtidos, a impossibilidade de diminuir o número de indicadores críticos que compõem o instrumento, uma vez que a análise por componentes principais demonstra que todos os indicadores são importantes, tendo cada um papel próprio na determinação do grau de complexidade do paciente em relação à atenção de enfermagem.

Uma variável (indicador crítico) somente pode ser eliminada quando estiver fortemente associada aos fatores que têm menor explicação na variação total e fracamente associada aos fatores mais importantes, isto é, com maior poder de explicação. Nesse caso, tem-se o fator 13 que é o que possui menor capacidade de explicação da variação total $(0,7 \%)$. A variável mais associada a esse fator é o indicador 12 (Comunicação). Se examinarmos o envolvimento dessa variável no fator 1 (de maior poder de explicação) podemos observar que ela está fortemente associada ao primeiro fator, devendo, portanto, ser mantida. A seguir, apresentamos, na Figura 1, a explicação dos fatores 1 e 2 através de diagrama de dispersão.

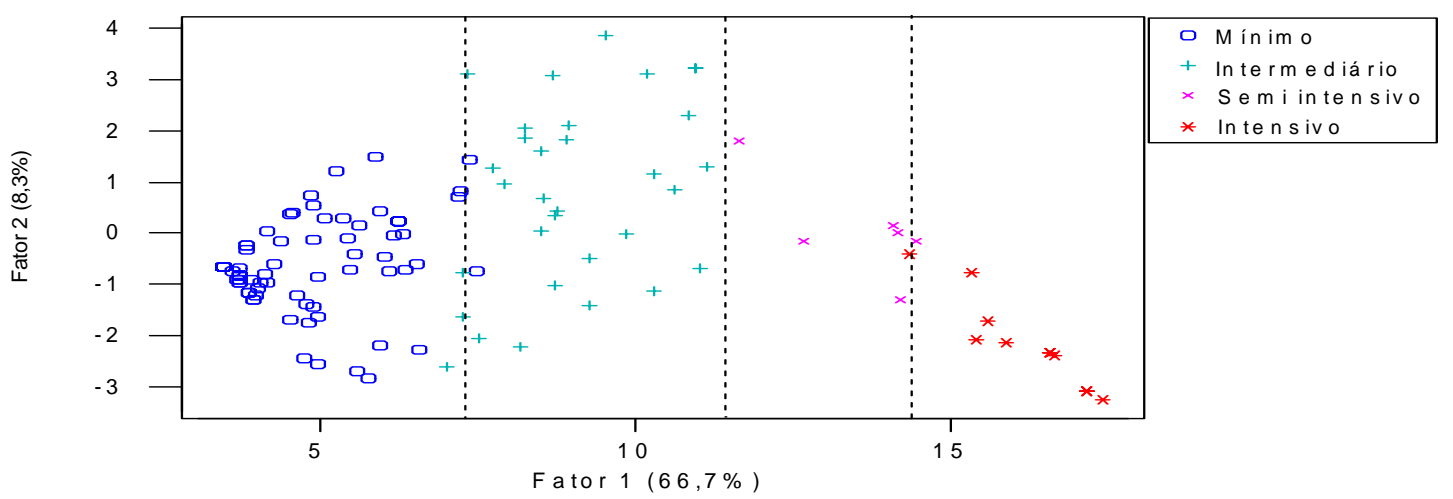

Figura 1 - Diagrama de dispersão dos pacientes em relação aos fatores 1 e 2, segundo o grau de complexidade. São José do Rio Preto, SP, 2000

Pode-se observar, pela Figura 1, a significância do fator 1 como discriminador das categorias de cuidados. Esse fator separa os pacientes em categorias (mínimos, intermediários, semi-intensivos e intensivos). Apesar de existirem sobreposições em algumas regiões, de maneira geral, o gráfico aponta diferenciação nítida entre as 
diferentes categorias de cuidados com valores crescentes à medida que a complexidade do cuidado aumenta.

Enquanto o fator 1 aponta nítida diferenciação entre as categorias de cuidado, o que é corroborado pela Análise de Variância na Tabela 2, o fator 2 mostra o que ocorre internamente em cada uma das categorias. Assim, a maior dispersão, em relação ao fator 2 é dada pelo grupo de pacientes classificados na categoria de cuidado intermediário. O grupo de pacientes classificados na categoria de intensivo é aquele que se apresenta mais homogêneo, provavelmente porque os escores obtidos em cada um dos indicadores são muito semelhantes e elevados. Ele é o grupo que apresenta menor contradição interna. Inversamente, o grupo que apresenta maior heterogeneidade e, portanto, com maiores diferenças individuais nas classificações, é o formado pela categoria de cuidados intermediários.

$\mathrm{Na}$ categoria de cuidado semi-intensivo, o fator 2 é pouco consistente, talvez pelo número reduzido de pacientes classificados nessa categoria, apenas seis. No fator 2 também se percebe, por meio de valores médios, diferenças significantes entre os grupos de cuidados mínimos, intermediários e intensivos (Tabela 3), indicando que os efeitos de contraposição dos grupos dos indicadores Motilidade, Locomoção, Cuidado Corporal e Eliminações e dos indicadores Estado Mental e Nível de Consciência, Oxigenação, Educação à Saúde, Comportamento, Comunicação e Integridade CutâneoMucosa são diferentes nesses graus de complexidade.

Tabela 2 - Análise de Variância do Fator 1. São José do Rio Preto, SP, 2000

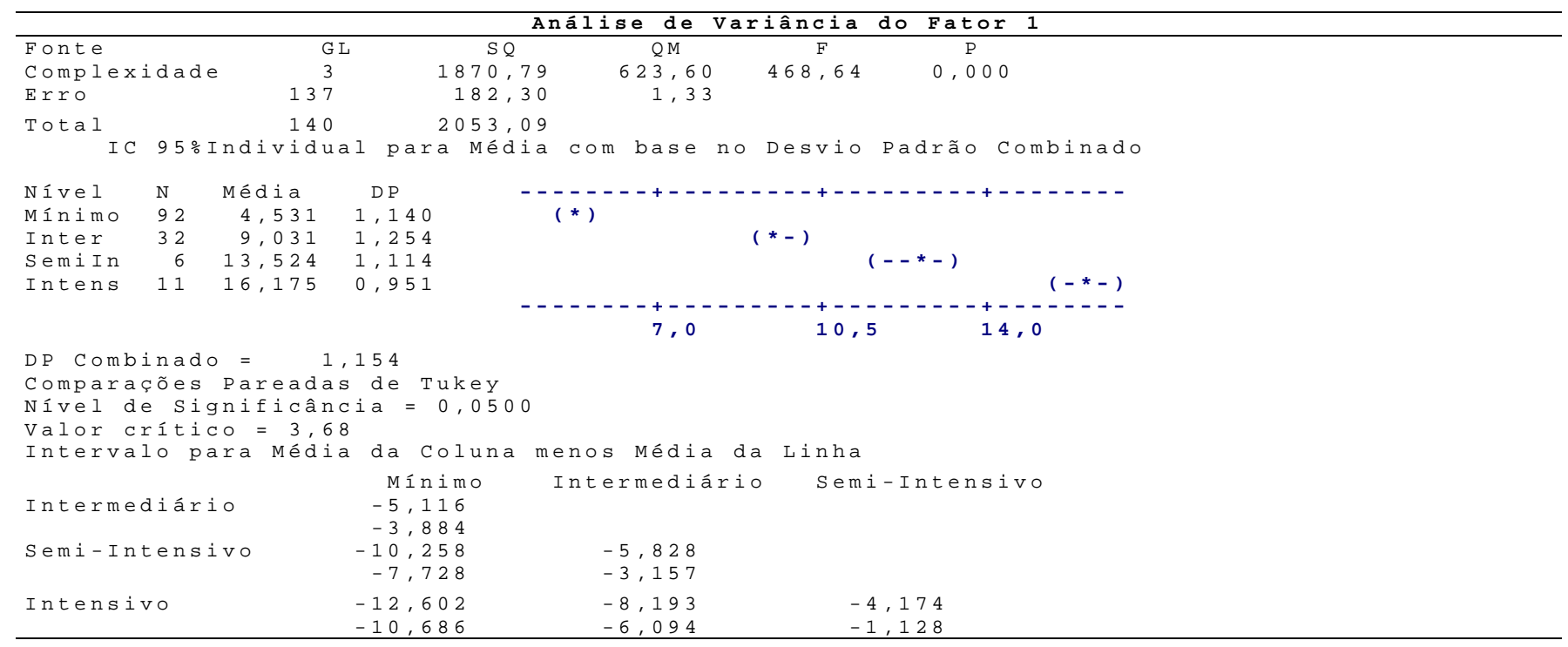

Tabela 3 - Análise de Variância do Fator 2. São José do Rio Preto, SP, 2000

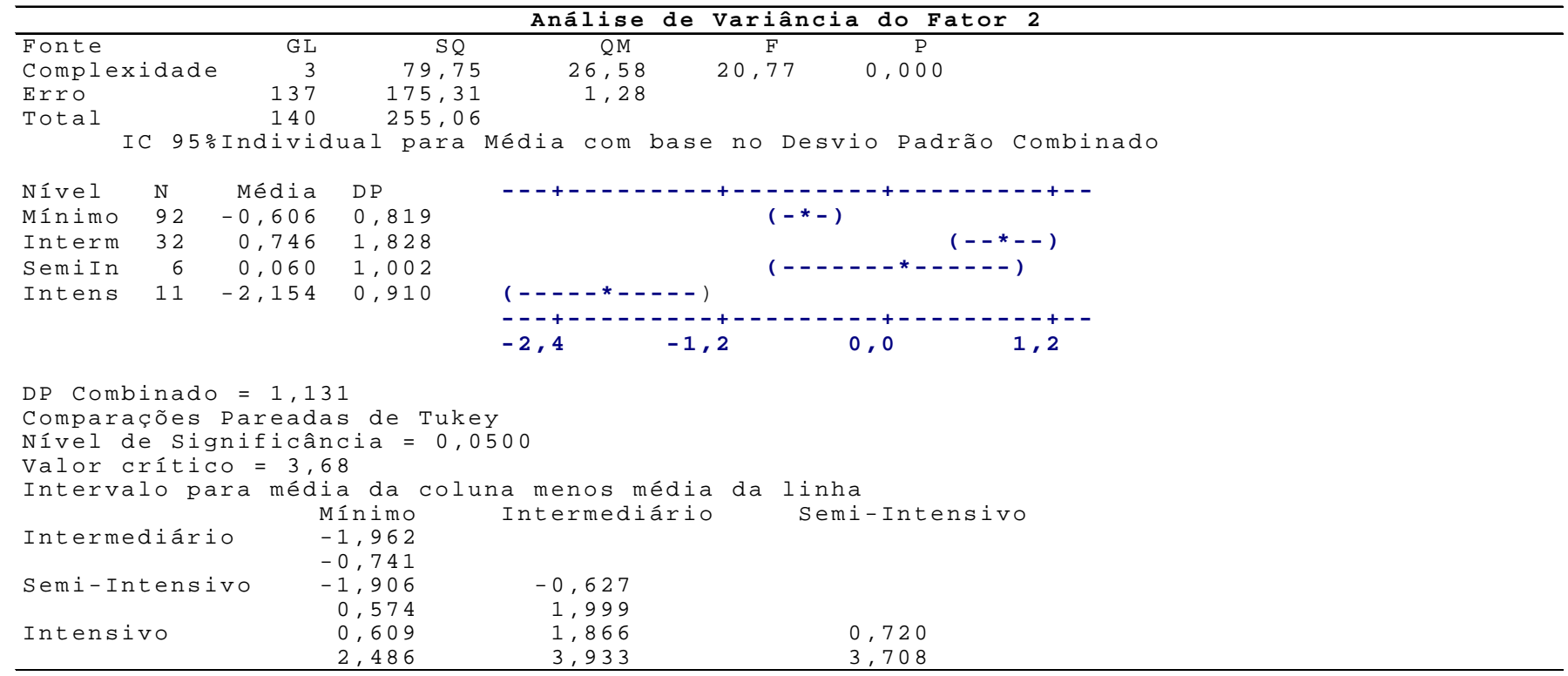


Analisando-se conjuntamente os dados das Tabelas 2 e 3, nota-se que o valor $\mathbf{p}$ abaixo de 0,05 indica que as 4 médias obtidas em cada uma das categorias de cuidados não são iguais, e as comparações pareadas pelo Método de Tukey indicam que as verdadeiras médias avaliadas pelo fator 1 seguem a ordenação: mínimos < intermediários < semi-intensivos < intensivos, no nível de significância $\alpha=0,05$.

O gráfico lateral à análise, na Tabela 3, ilustra esse efeito no qual se observa o valor médio do fator em cada um dos níveis de cuidado. A existência de uma ordenação torna possível interpretar o fator 1 como "grau de complexidade", uma vez que ele cria um gradiente de complexidade diferenciando os níveis de cuidados mínimos, intermediários, semi-intensivos e intensivos.

Estudo das Correlações (Pearson) entre os indicadores críticos

Considerando-se os 13 indicadores que compõem o instrumento de classificação de pacientes de Perroca ${ }^{(1)}$, obteve-se correlação positiva entre todos eles com Pearson variando de 0,309 a 0,882. Isso evidencia a validade de construção do instrumento, mostrando coerência interna do mesmo. Os coeficientes de correlação Pearson encontrados podem ser evidenciados na Tabela 4.

Tabela 4 - Coeficiente de Correlação de Pearson entre os 13 indicadores que compõem o instrumento de classificação de pacientes. São José do Rio Preto, SP, 2000

\begin{tabular}{|c|c|c|c|c|c|c|c|c|c|c|c|c|}
\hline & Ind 1 & Ind 2 & Ind 3 & Ind 4 & Ind 5 & Ind 6 & Ind 7 & Ind 8 & Ind 9 & Ind 10 & Ind 11 & Ind 12 \\
\hline Ind 2 & 0,7188 & & & & & & & & & & & \\
\hline Ind 3 & 0,403 & 0,490 & & & & & & & & & & \\
\hline Ind 4 & 0,727 & 0,663 & 0,493 & & & & & & & & & \\
\hline Ind 5 & 0,669 & 0,619 & 0,351 & 0,6890, & & & & & & & & \\
\hline Ind 61 & 0,579 & 0,539 & 0,416 & 0,684 & 0,842 & & & & & & & \\
\hline Ind 7 & 0,644 & 0,549 & 0,421 & 0,725 & 0,822 & 0,882 & & & & & & \\
\hline Ind 8 & 0,628 & 0,600 & 0,459 & 0,763 & 0,790 & 0,851 & 0,849 & & & & & \\
\hline Ind 9 & 0,506 & 0,632 & 0,534 & 0,686 & 0,587 & 0,640 & 0,580 & 0,667 & & & & \\
\hline Ind 10 & 0,702 & 0,570 & 0,401 & 0,5950 & 0,493 & 0,484 & 0,550 & 0,522 & 0,510 & & & \\
\hline Ind 11 & 0,688 & 0,583 & 0,401 & 0,596 & 0,527 & 0,558 & 0,591 & 0,560 & 0,534 & 0,580 & & \\
\hline Ind 12 & 0,850 & 0,742 & 0,341 & 0,779 & 0,709 & 0,645 & 0,710 & 0,697 & 0,533 & 0,605 & 0,752 & \\
\hline Ind 13 & 0,552 & 0,470 & 0,309 & 0,536 & 0,476 & 0,496 & 0,475 & 0,451 & 0,444 & 0,497 & 0,544 & 0,541 \\
\hline
\end{tabular}

Os indicadores mais correlacionados foram: 1Locomoção com os indicadores: Cuidado Corporal $(0,882)$, Eliminações $(0,851)$ e Motilidade $(0,842) ; 2$ - Cuidado Corporal com os indicadores: Eliminações $(0,849)$ e Motilidade e 3- Estado Mental e Nível de Consciência com o indicador Comunicação $(0,850)$, enquanto o indicador que apresentou menor correlação foi Sinais Vitais com os indicadores Integridade Cutâneo-Mucosa $(0,309)$, Comunicação $(0,341)$ e Motilidade $(0,351)$.

Essas elevadas correlações positivas entre os indicadores Locomoção, Cuidado Corporal e Eliminações mostra que essas variáveis estão fortemente atreladas, de modo que o aumento da dependência do paciente em relação ao cuidado de enfermagem em um desses indicadores conseqüentemente levará ao aumento dos outros dois. Isso é fácil de ser entendido, uma vez que o paciente que pode movimentar-se dentro do ambiente físico, por si só, também poderá realizar suas atividades de higiene pessoal e o controle de suas eliminações. De outra forma, se ele se encontra acamado estará impossibilitado de realizar por si mesmo atividades de higiene pessoal, de conforto, de vestir-se e arrumar-se, requerendo auxílio do acompanhante, ou da enfermagem, tanto para esse cuidado, como também para manter as diversas formas de eliminações corporais. O mesmo raciocínio pode ser utilizado para se explicar a correlação direta entre os indicadores Cuidado Corporal e Eliminações.

Contudo, a alta relação entre os indicadores Locomoção e Motilidade e Cuidado Corporal e Motilidade remete-nos à seguinte reflexão: se considerarmos motilidade como a capacidade do paciente movimentar 
os segmentos corporais de forma independente, com auxílio do acompanhante, ou da equipe de enfermagem, ou pelo uso de artefatos, fica claro que se o paciente deambula ele é capaz de movimentar todos os seus segmentos e do autocuidado para higiene corporal. Entretanto, a impossibilidade de locomoção, ou de realização do cuidado corporal por si mesmo, não conduz necessariamente à incapacidade de movimentação dos segmentos. Isso pode ocorrer apenas em algumas situações quando há déficit motor, ou quando o paciente é portador de aparelhos gessados, fixador externo, tração, ou outros.

Nesses achados é encontrada, também, forte correlação entre o indicador Estado Mental e Nível de Consciência com o indicador Comunicação. A efetividade do processo de comunicação na interação humana depende da habilidade em se manter a percepção e as atividades cognitivas. $O$ indicador Sinais Vitais foi o que apresentou menor correlação com todos os demais indicadores. Sua maior correlação foi com o indicador Terapêutica $(0,534)$, o que pode ser explicado devido à necessidade de observação e controle cada vez mais rigoroso dos parâmetros vitais à medida que se faz uso de agentes terapêuticos medicamentosos que exijam maiores cuidados de enfermagem na administração.

\section{CONCLUSÕES E IMPLICAÇÕES PARA A PRÁTICA DE ENFERMAGEM}

Antecedendo à implementação de um instrumento na prática assistencial, torna-se imperativo viabilizar a demonstração de seu grau de confiabilidade e validade a fim de que as informações fornecidas por ele possam ser utilizadas com confiança pelo serviço de enfermagem.

Considerando-se que a validação é um processo

\section{REFERÊNCIAS BIBLIOGRÁFICAS}

1. Perroca MG.Sistema de classificação de pacientes: construção e validação de um instrumento. [Dissertação]. São Paulo (SP): Escola de Enfermagem/USP; 1996.

2. Perroca MG, Gaidzinski RR. Sistema de classificação de pacientes: construção e validação de um instrumento. Rev Esc Enfermagem USP 1998; 32(2):153-68.

3. Nunnally JC. Validity. In: Nunnally, JC. Psychometric theory. New York: Mc Graw-Hill; 1967. p.75-102.

4. Kerlinger FN. Observação e mensuração de variáveis. In: Kerlinger FN. Metodologia da pesquisa em ciências sociais. São Paulo: EPU; 1980. p. 144-61. infinito e também que a validade de um instrumento não é provada, mas apoiada em um grau maior ou menor de evidências, podemos dizer que o instrumento de classificação de Perroca ${ }^{(1)}$ demonstra evidências suficientes de confiabilidade e validade, podendo ser utilizado como instrumento diagnóstico de categoria de cuidado a que o paciente pertence.

A análise das associações entre os 13 indicadores críticos, construídos para compor o instrumento, evidencia correlações positivas entre todos os indicadores críticos, tendo cada um deles, individualmente, sua importância na estrutura global do instrumento, embora alguns tenham apresentado maior importância do que outros. Os achados revelam a não existência de indicadores compulsórios para uma determinada categoria de cuidados, mas, sim, a presença de indicadores mais significativamente importantes para captar a complexidade do cuidado. $\mathrm{O}$ indicador 7 (Cuidado Corporal) é apontado como o mais importante para captar essa mudança, enquanto o indicador 3 (Sinais Vitais) é aquele que apresenta menor importância. Dessa forma, torna-se inviável a redução do número de indicadores críticos no instrumento de classificação de pacientes, uma vez que eles atuam conjuntamente para discriminar o grau de complexidade das necessidades do paciente.

Diante desses resultados, este estudo permitiu concluir que o instrumento de classificação de pacientes de Perroca ${ }^{(1)}$ apresenta condições de ser aplicado na prática gerencial do enfermeiro como norteador das necessidades de cuidado do paciente, bem como da carga de trabalho da equipe de enfermagem. Novos testes, junto a diferentes perfis de clientela da população deste estudo, são recomendados a fim de que o instrumento seja refinado. $O$ instrumento pode servir, ainda, como base para posteriores pesquisas com o intuito de aprimorar 0 processo de cuidar em enfermagem.

5. Waltz C, Bausell RB. Measurement. In: Waltz C, Bausell RB. Nursing research: design, statistics and computer analysis. Philadelphia: F.A. Davis; 1981. p. 39-82.

6 . Keeves JP. Measurement for educational research. In: Keeves, JP. Educational research, methodology and measurement. 2nd ed. Great Britain: Pergamon Press;1990. p. 322-30.

7. Polit DF, Hungler BP. Análise Quantitativa. In: Polit DF, Hungler BP. Fundamentos de pesquisa em enfermagem. 3 ed. Porto Alegre: Artes Médicas; 1995. p. 223-67.

8. Kerlinger FN. Validity. In: Kerlinger FN. Foundations of behavioral research. New York: Holt, Rinehart and Winston; 1964. p. 444-62.

9. Johnson RA, Wichern DW. Applied multivariate statistical analysis. Englewood Cliffs: Prentice Hall; 1992. 
10. Cuadras CM. Análisis de componentes principais. In: Cuadras CM. Métodos de análisis multivariante. Barcelona: Editorial Universitária de Barcelona; 1981. p. 269-93.

11. Wonnacott TH, Wonnacott RJ. Análise da Variância. In: Wonnacott TH, Wonnacott RJ. Estatística aplicada à economia e à administração. Rio de Janeiro: Livros Técnicos e Científicos; 1981. p. 247-80.

12. Pavani LMD. Análise do dimensionamento de pessoal de enfermagem na unidade de pós- operatório de cirurgia cardíaca em um hospital universitário especializado em cardiologia. [Dissertação]. São Paulo (SP): Escola de Enfermagem/USP; 2000. 